\title{
MS43-P02 | Instrumental Effects in Laboratory Pair Distribution Function (PDF)
}

\section{ANALYSIS.}

Gateshki, Milen (Malvern Panalytical B.V., Almelo); Beckers, Detlef (Malvern Panalytical B.V., Almelo); Kogan, Vladimir (Dannalab B.V., Enschede)

The PDF technique utilizes a Fourier transformation of the X-ray powder diffraction (XRPD) data and gives information about the inter-atomic distances of the material. The accuracy of this determination of inter-atomic distances depends strongly on the energy of the utilized radiation source and the ability to correct for samplerelated effects and for aberrations from the instrumental set-up that is used. Recent advances in laboratory X-ray diffractometer technology e.g. new generation detectors optimized for hard radiation (e.g. Mo and Ag radiation) allow to minimize artifacts or fluctuations in the PDF arising from statistical noise, resulting in more reliable data. Nevertheless, effects of instrumental aberrations such as axial divergence or spectral features of the X-ray source may still influence the accuracy of the final result.

In this contribution we evaluate the effect of different instrumental parameters on the calculated PDF and discuss possible ways to minimize the influence of instrumental aberrations. In the specific case of axial divergence this can be done by using narrow Soller slits, which necessarily reduce the intensity of the measured scattering signal or by applying a data correction procedure before the PDF is calculated. This procedure [1, 2] can correct for systematic instrumental aberrations while considering multiple overlapping peaks as a single continuum. It is based on an integral transformation algorithm and converts the measured pattern into the corrected one without influencing the angular resolution of the measurement.

[1] Kogan V.A., Kupriyanov M.F., J.Appl.Cryst, 1992, 25,16-2

[2] Kogan V.A. US7516031B2 "Apparatus and method for correcting for aberrations" 